\title{
REFERENCES
}

1. P. E. Blanksby and H. L. Montgomery, Algebraic integers near the unit circle, Acta Arith. 18 (1971), 355-369. MR 45\#5082.

2. S. Chowla, On polynomials all of whose roots lie on the unit circle, J. Reine Angew. Math. 222 (1966), 69-70. MR 32 \#5604.

3. L. Kronecker, Zwei Sätze über Gleichungen mit ganzzahligen Coefficienten, J. Reine Angew. Math. 53 (1857), 173-175.

4. Paul Turán, Eine neue Methode in der Analysis und deren Anwendungen, Akad. Kiadó, Budapest, 1953. MR 15, 688.

5. A. J. van der Poorten, Generalisations of Turán's main theorems on lower bounds for sums of powers, Bull. Austral. Math. Soc. 2(1970), 15-37. MR 42 \#217.

DEPARTMENT OF PURE MATHEMATICS, UNIVERSITY OF ADELAIDE, ADELAIDE, SOUTH AUSTRALIA, AUSTRALIA 5001

PROCEEDINGS OF THE

AMERICAN MATHEMATICAL SOCIETY

Volume 49, Number 1, May 1975

\section{THE DIMENSION OF THE RING OF COEFFICIENTS IN A POLYNOMIAL RING}

\author{
JIMMY T. ARNOLD
}

ABSTRACT. $A$ and $B$ are commutative rings with identity. We say that $A$ and $B$ are stably equivalent provided there exists a positive integer $n$ such that the polynomial rings $A\left[X_{1}, \cdots, X_{n}\right]$ and $B\left[Y_{1}, \cdots, Y_{n}\right]$ are isomorphic. If $A$ and $B$ are stably equivalent, then they have equal Krull dimen sion.

The question answered in this paper arises from recent investigations concerning the uniqueness of the ring of coefficients in a polynomial ring (cf. [1]-[6]). In [6], Hochster has given an example which illustrates that stably equivalent rings need not be isomorphic. Several related questions are posed by Eakin and Heinzer in [5]. In particular, if $A$ and $B$ are stably equivalent rings, then Eakin and Heinzer ask whether $\operatorname{dim} A=\operatorname{dim} B(\operatorname{dim} R$ denotes the Krull dimension of the ring $R$ ). We shall presently show that this

Received by the editors February 1, 1974.

AMS (MOS) subject classifications (1970). Primary 13B25; Secondary 13A15, $13 \mathrm{C} 15$.

Key words and phrases. Polynomial ring, Krull dimension, coefficient ring. 
is indeed the case. Our proof is based on the following well-known result.

(A) If $P$ is a prime ideal of the ring $R$ and if $Q_{1} \subset \cdots \subset Q_{k}$ is a chain of $k$ distinct prime ideals of the polynomial ring $R\left[X_{1}, \cdots, X_{m}\right]$ such that $Q_{i} \cap R=P$ for each $i$, then $k \leq m+1$.

This result is the natural generalization of Theorem 37 of [7] and can be proved in a similar fashion.

Theorem. If $A$ and $B$ are stably equivalent, then $\operatorname{dim} A=\operatorname{dim} B$.

Proof. We assume without loss of generality that $A[X]=B[Y]$, where $X=\left\{X_{i}\right\}_{i=1}^{m}$ and $Y=\left\{Y_{i}\right\}_{i=1}^{m}$ are indeterminates over $A$ and $B$, respectively. It suffices to consider the case in which $A$ and $B$ are integral domains and since the result clearly holds if $A$ has infinite dimension, we assume that $\operatorname{dim} A=n$ is finite. If $n=0$, then $\operatorname{dim} A=\operatorname{dim} B ;$ in fact, $A=B$ (since the units of $A[X]=B[Y]$ are precisely the units of $A$ (or $B)$ ). Thus, we suppose that $n \geq 1$ and we show that $\operatorname{dim} B \geq n$. This is clear if $n=1$, so assume that $n \geq 2$ and let (0) $\subset P_{1} \subset \cdots \subset P_{n}$ be a maximal chain of prime ideals of $A$. For a subset $S$ of $A$ we shall let $\chi_{m} S$ denote the set of $m$-tuples of elements of $S$. If $a=\left(a_{1}, \cdots, a_{m}\right) \in X_{m} A$, then for $1 \leq k \leq n$ we let $p_{k}^{(a)}$ denote the prime ideal $\left(P_{k}, X_{1}+a_{1}, \cdots, X_{m}+a_{m}\right)$ of $A[X]$ and we set $Q_{k}^{(\alpha)}=P_{k}^{(\alpha)} \cap B$. In particular, for $k=1$ we get the chain

(0) $\subset P_{1}[X] \subset\left(P_{1}, X_{1}+a_{1}\right) \subset \ldots \subset\left(P_{1}, X_{1}+a_{1}, \cdots, X_{m-1}+a_{m-1}\right) \subset P_{1}^{(a)}$

of $m+2$ distinct prime ideals of $B[Y]$. It follows from (A) that $Q_{1}^{(\alpha)}=P_{1}^{(a)}$ $\cap B \neq(0)$, that is, rank $Q_{1}^{(a)} \geq 1$.

Obviously our proof is complete if we can show the existence of an element $\alpha$ in $X_{m} A$ such that $\operatorname{rank} Q_{k}^{(\alpha)} \geq k$ for each $k, 1 \leq k \leq n$. Therefore, suppose that no such $\alpha$ exists. Then we may choose a smallest integer $t$ for which there exists an element $\alpha_{0}$ in $\mathrm{X}_{m} A$ such that rank $Q_{t}^{\left(a_{0}\right)}<t$. We have already observed that $t a_{\left(a_{0}+\beta\right)}$. Set $\mathcal{S}=X_{m}\left(P_{t}-P_{t-1}\right)$ and let $\beta \in \mathcal{S}$. It is clear that $P_{t}^{\left(a_{0}+\beta\right)}=P_{t}^{\left(\alpha_{0}\right)}$ (where $\alpha_{0}+\beta$ is defined in the usual way), so we have

$$
Q_{t}^{\left(\alpha_{0}\right)}=Q_{t}^{\left(\alpha_{0}+\beta\right)}=P_{t}^{\left(\alpha_{0}+\beta\right)} \cap B \supseteq P_{t-1}^{\left(\alpha_{0}+\beta\right)} \cap B=Q_{t-1}^{\left(\alpha_{0}+\beta\right)} .
$$

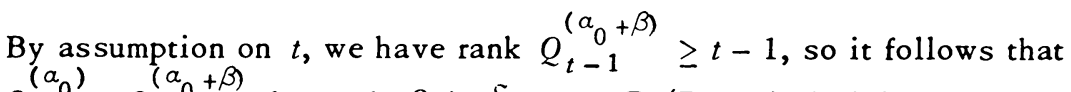
$Q_{t}^{\left(a_{0}\right)}=Q_{t-1}^{\left(a_{0}+\beta\right)}$ for each $\beta$ in $\delta$. Now $P_{t} / P_{t-1}$ is infinite, so for any $a$ in $A$, the set $\left\{a+p \mid p \in P_{t}-P_{t-1}\right\}$ contains infinitely many elements which are distinct modulo $P_{t-1}$. Therefore, 


$$
P_{t-1}[X]=\bigcap_{\beta \in \mathcal{S}} P_{t-1}^{\left(a_{0}+\beta\right)} \supseteq \bigcap_{\beta \in \mathcal{S}} Q_{t-1}^{\left(\alpha_{0}+\beta\right)}[Y]=Q_{t}^{\left(\alpha_{0}\right)}[Y] .
$$

If $a_{0}=\left(a_{1}, \cdots, a_{m}\right)$, then we have a chain

$$
\begin{aligned}
Q_{t}^{\left(a_{0}\right)}[Y] & \subseteq P_{t-1}[X] \subset\left(P_{t-1}, X_{1}+a_{1}\right) \subset \cdots \\
& \subset\left(P_{t-1}, X_{1}+a_{1}, \cdots, X_{m-1}+a_{m-1}\right) \subset P_{t-1}^{\left(a_{0}\right)} \subset P_{t}^{\left(a_{0}\right)}
\end{aligned}
$$

of at least $m+2$ prime ideals of $B[Y]$ all of which contract to $Q_{t}^{\left(a_{0}\right)}$ in $B$. This contradicts $(A)$, so our proof is complete.

\section{REFERENCES}

1. S. Abhyankar, P. Eakin and W. Heinzer, On the uniqueness of the coefficient ring in a polynomial ring, J. Algebra 23 (1972), 310-342. MR 46 \#5300.

2. J. Brewer and E. Rutter, Isomorphic polynomial rings, Arch. Math. 23 (1972), 484-488.

3. D. B. Coleman and E. E. Enochs, Isomorphic polynomial rings, Proc. Amer. Math. Soc. 27 (1971), 247-252. MR $42 \# 7686$.

4. P. Eakin and K. Kubota, $A$ note on the uniqueness of rings of coefficients in polynomial rings, Proc. Amer. Math. Soc. 23 (1972), 333-341. MR 45 \#6815.

5. P. Eakin and W. Heinzer, A cancellation problem for rings, Proc. Conference on Commutative Algebra, Lecture Notes in Math., vol. 311, Springer-Verlag, Berlin and New York, 1973, pp. 61-77.

6. M. Hochster, Nonuniqueness of coefficient rings in a polynomial ring, Proc. Amer. Math. Soc. 34 (1972), 81-82. MR 45 \#3394.

7. I. Kaplansky, Commutative rings, Allyn and Bacon, Boston, Mass., 1970. MR 40 \#7234.

DEPARTMENT OF MATHEMATICS, VIRGINIA POLYTECHNIC INSTITUTE AND STATE UNIVERSITY, BLACKSBURG, VIRGINIA 24061 